\title{
Butein inhibits lipogenesis in Caenorhabditis elegans
}

$\underline{\text { Renalison Farias-Pereira }}^{a}$, Zhenyu Zhang ${ }^{a}$, Cheon-Seok Park ${ }^{b}$, Kee-Hong Kim ${ }^{c, d}$, and Yeonhwa Park ${ }^{a, b}$

a Department of Food Science, University of Massachusetts, Amherst, MA, 01003, USA

b Department of Food Science \& Biotechnology, Kyung Hee University, Yongin, 17104, Korea

d Department of Food Science, Purdue University, West Lafayette, IN, 47907, USA

e Center for Cancer Research, Purdue University, West Lafayette, IN, 47907, USA 


\section{What is Butein?}

- Flavonoid chalcone with potential therapeutic uses found in annatto seeds and lacquer trees

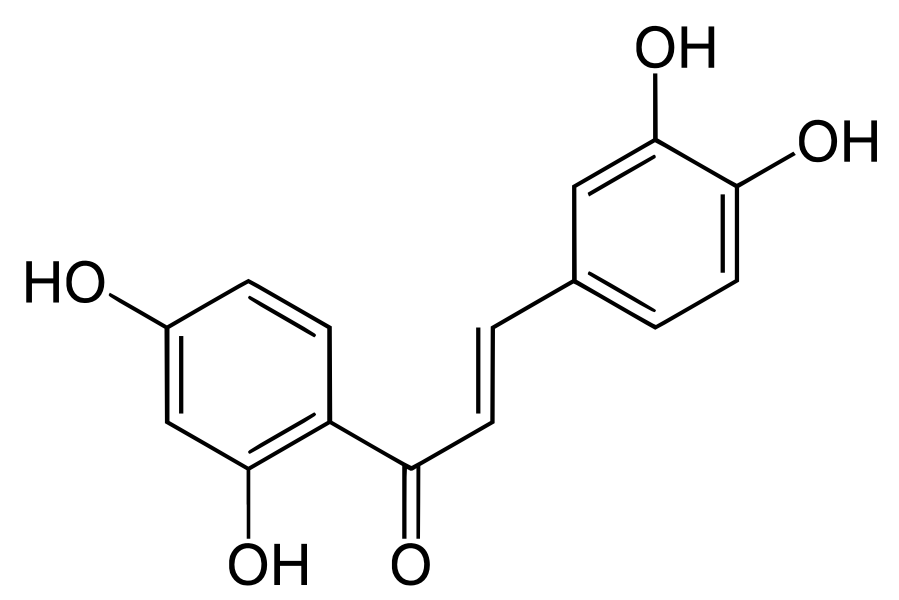




\section{Why Caenorhabditis elegans?}

- Nematode, eukaryotic, multi-organ animal model used in obesity research

- Different mutants are used to clarify mechanisms of action of bioactives on lipid metabolism 


\section{Butein reduced fat accumulation in $C$. elegans}

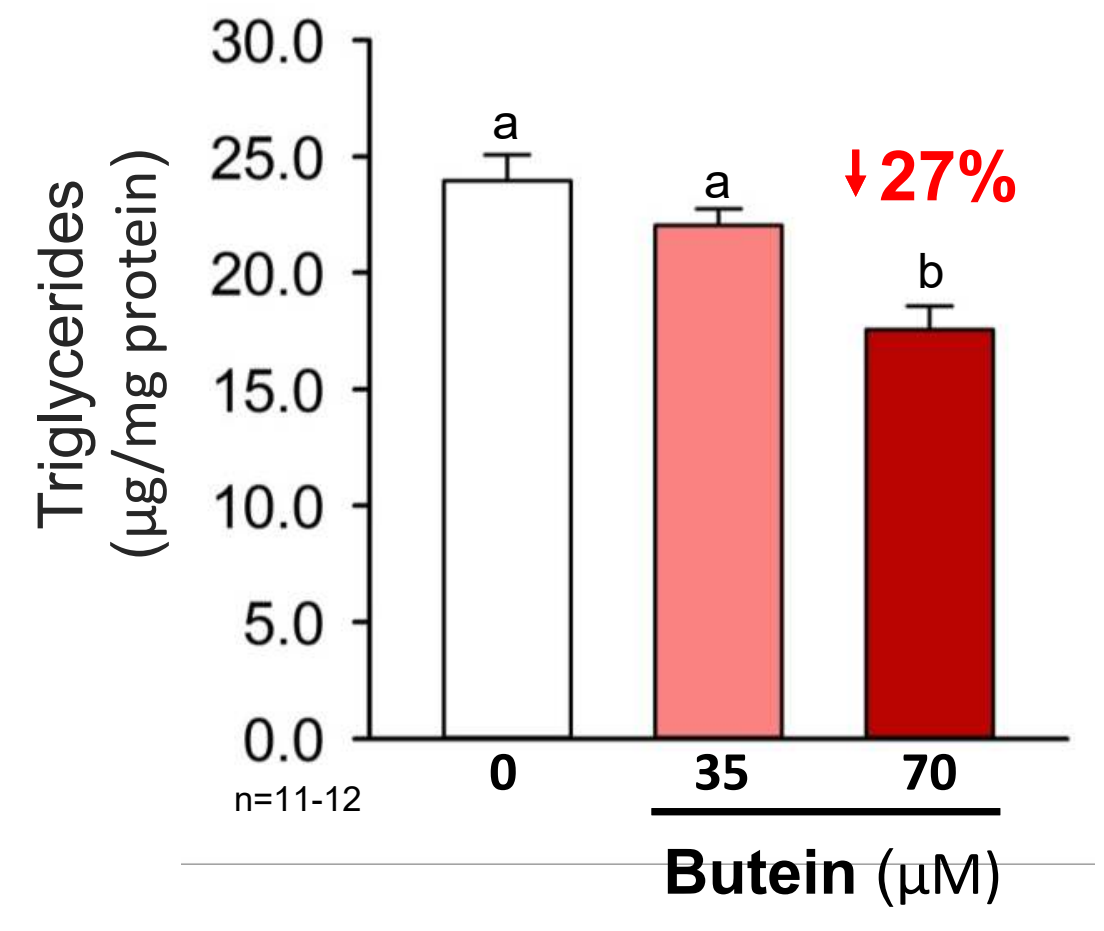




\section{Butein did not change pharynx pumping rate}

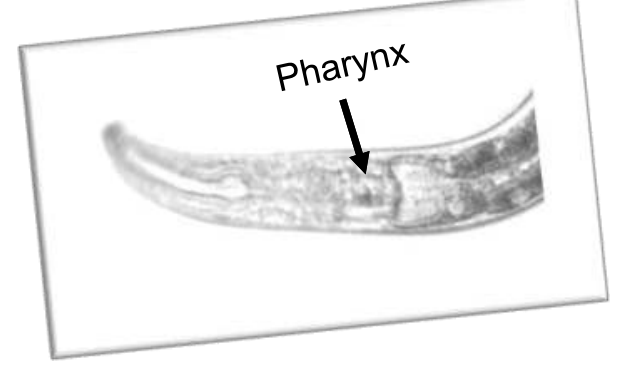

- Pharynx is the organ that pumps food to inside of $C$. elegans

- Pharynx pumping rate is an indicator of food intake

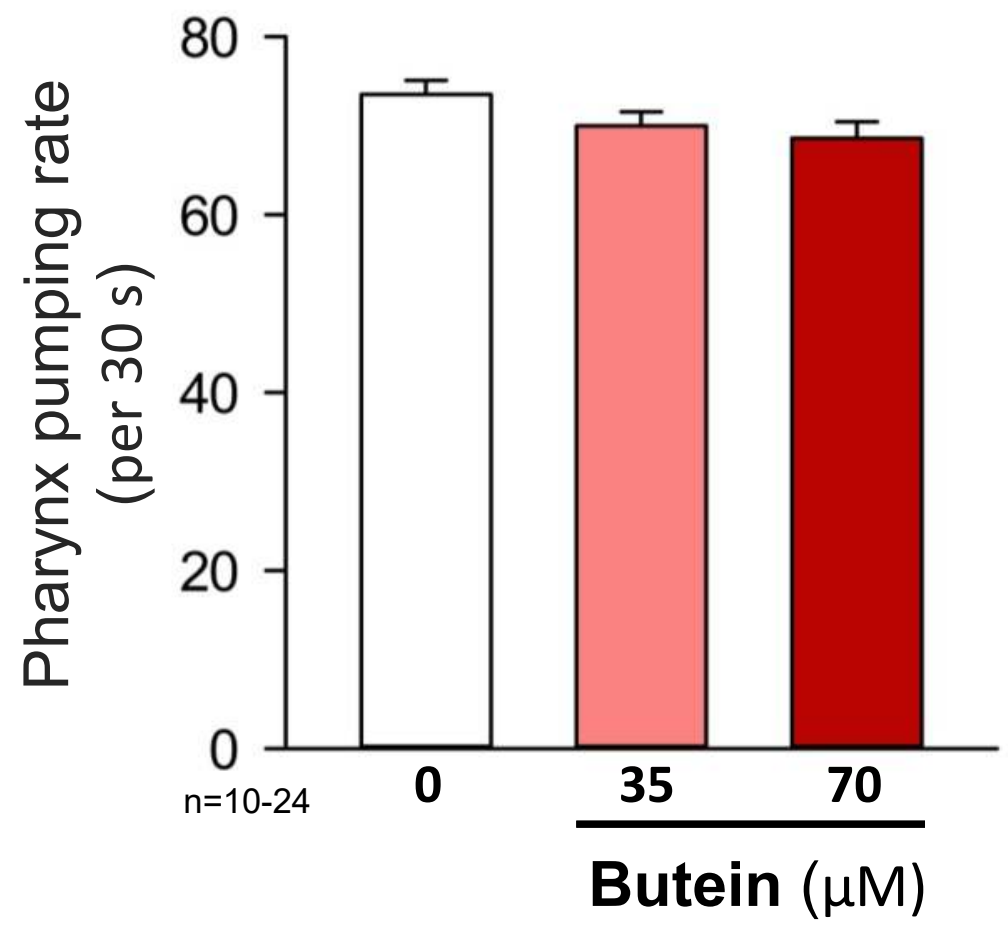




\section{Butein did not change moving speed of worms}

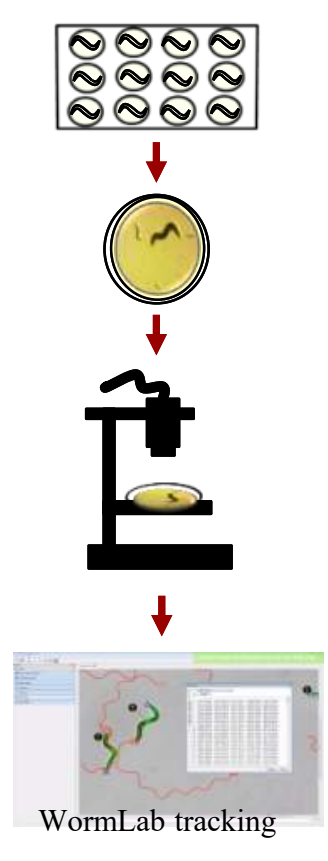

- Moving speed of worms is an indicator of energy expenditure

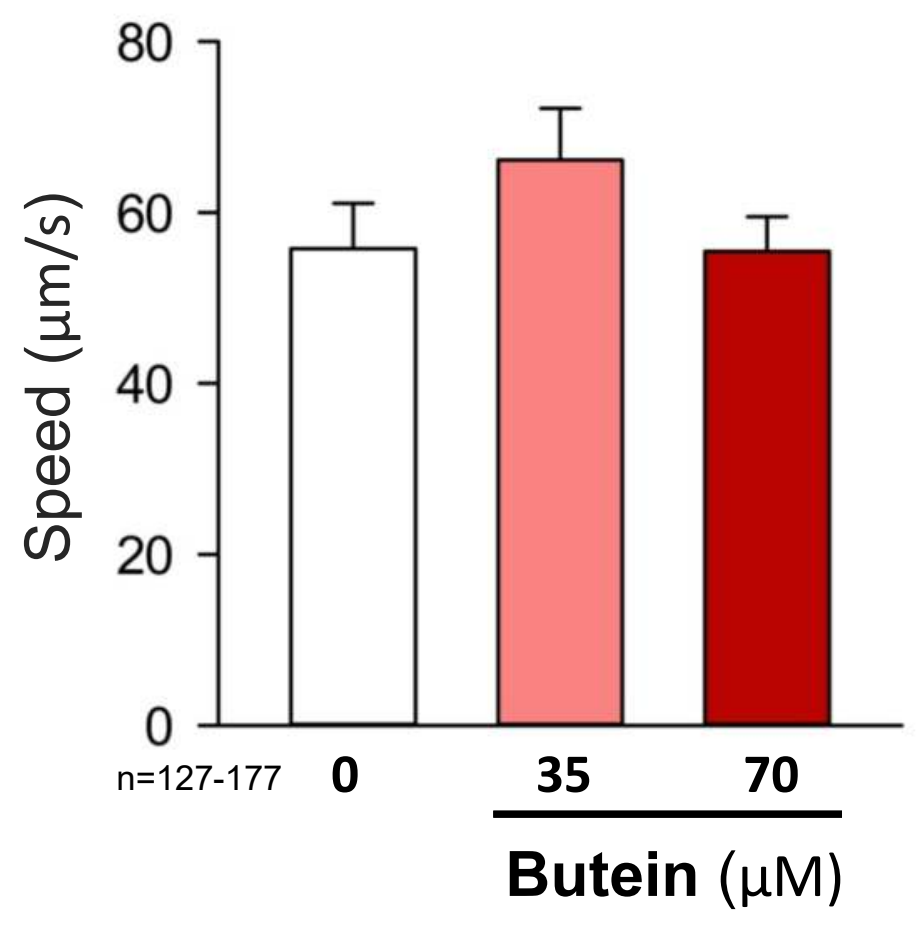




\section{The effects of butein on fat accumulation in different knockout mutants}

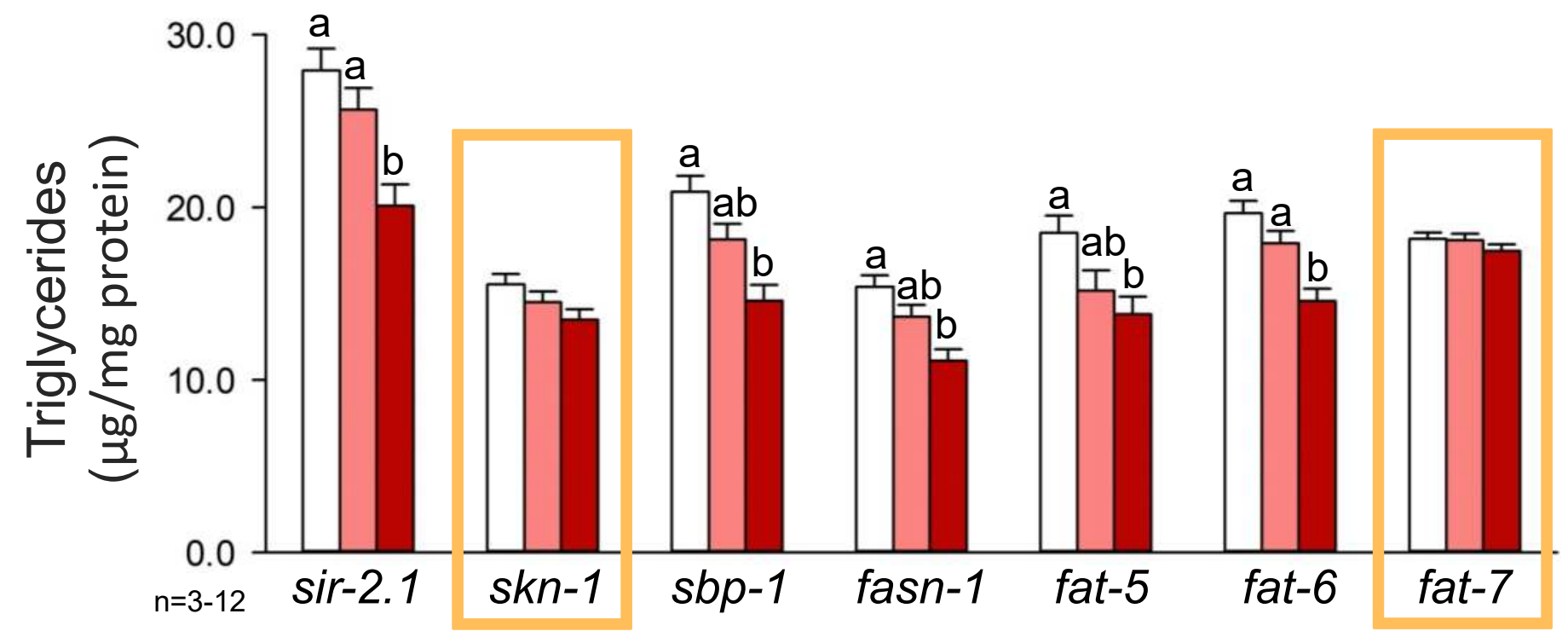

sir-2.1, homolog of SIRT1, involved in energy homeostasis skn-1, homolog of Nrf, involved in antioxidant pathways 


\section{Butein downregulated the lipogenesis-related genes: sbp-1, fasn-1 and fat-7}

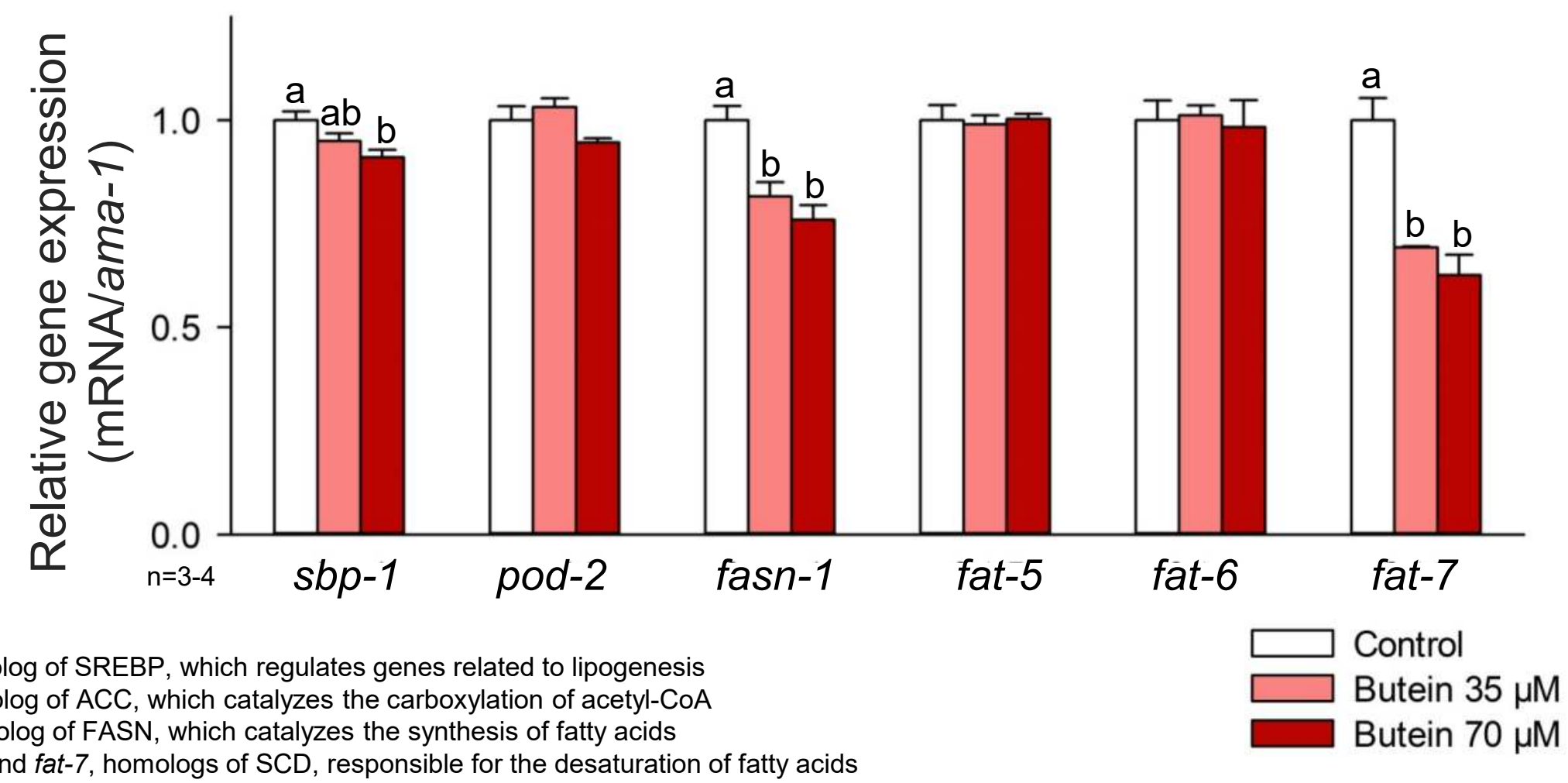

sbp-1, homolog of SREBP, which regulates genes related to lipogenesis pod-2, homolog of ACC, which catalyzes the carboxylation of acetyl-CoA fasn-1, homolog of FASN, which catalyzes the synthesis of fatty acids fat-5, fat- 6 and fat-7, homologs of SCD, responsible for the desaturation of fatty acids 


\section{Butein did not change fatty acids profile}

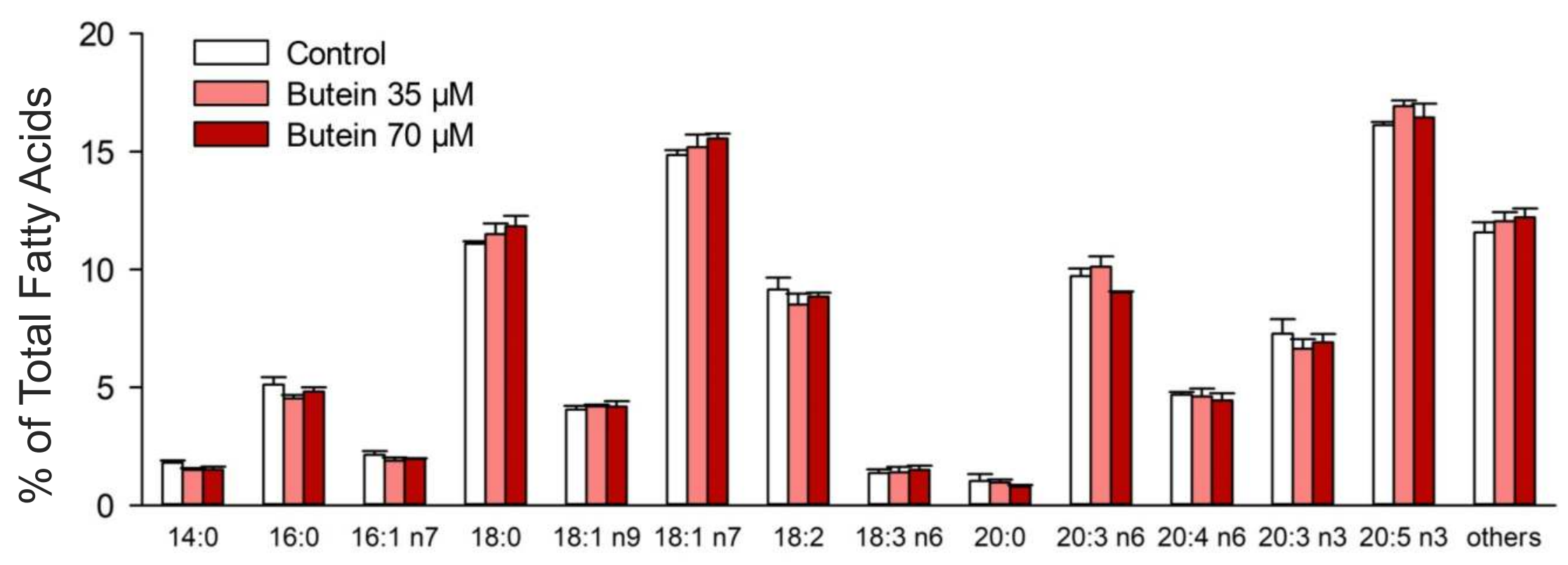




\section{Butein downregulated sbp-1 and fasn-1 via SKN-1-dependent pathway in C. elegans}

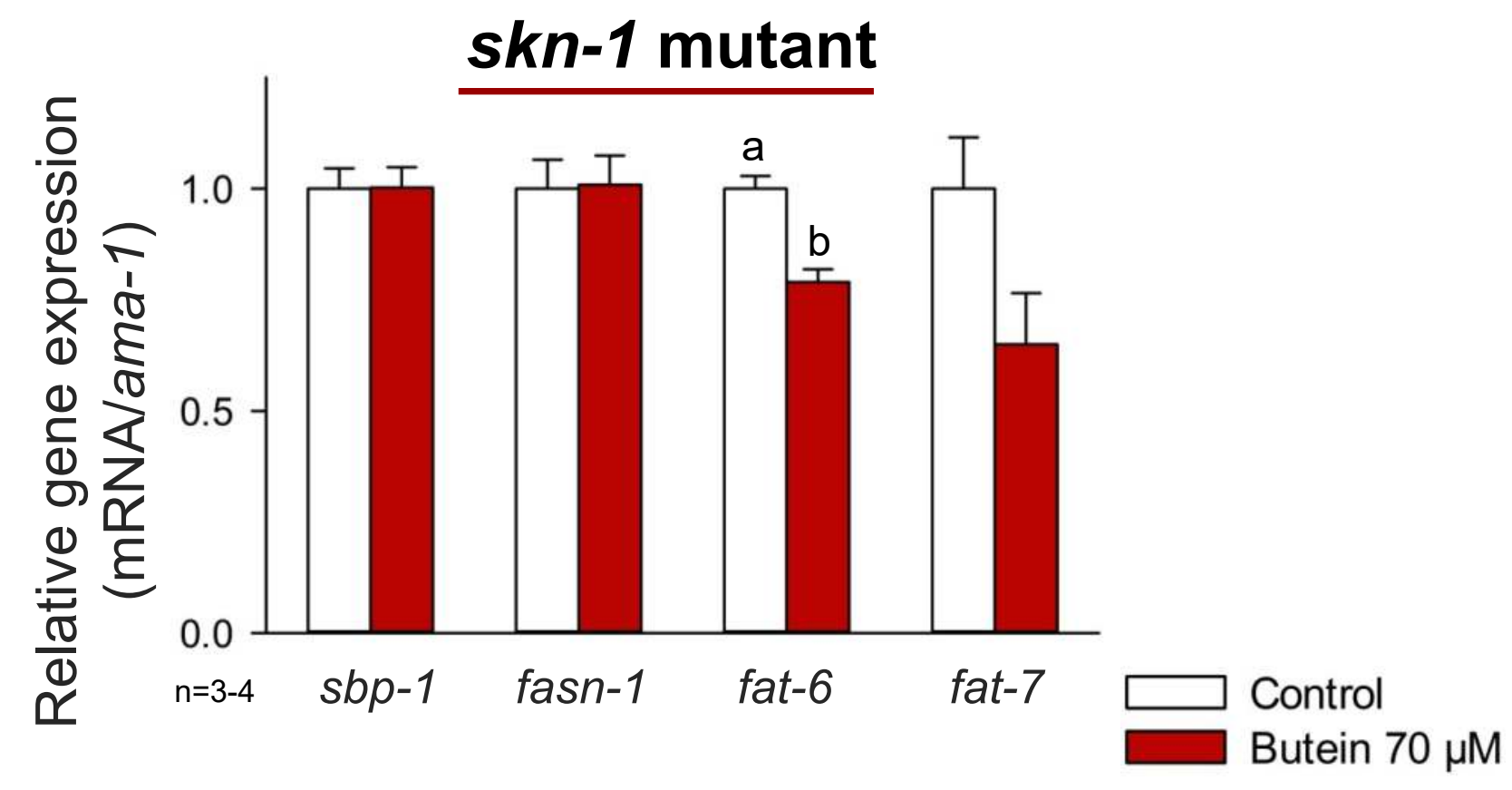




\section{Butein reduced fat accumulation}

\section{via SKN-1- and FAT-7-dependent pathways in C. elegans}

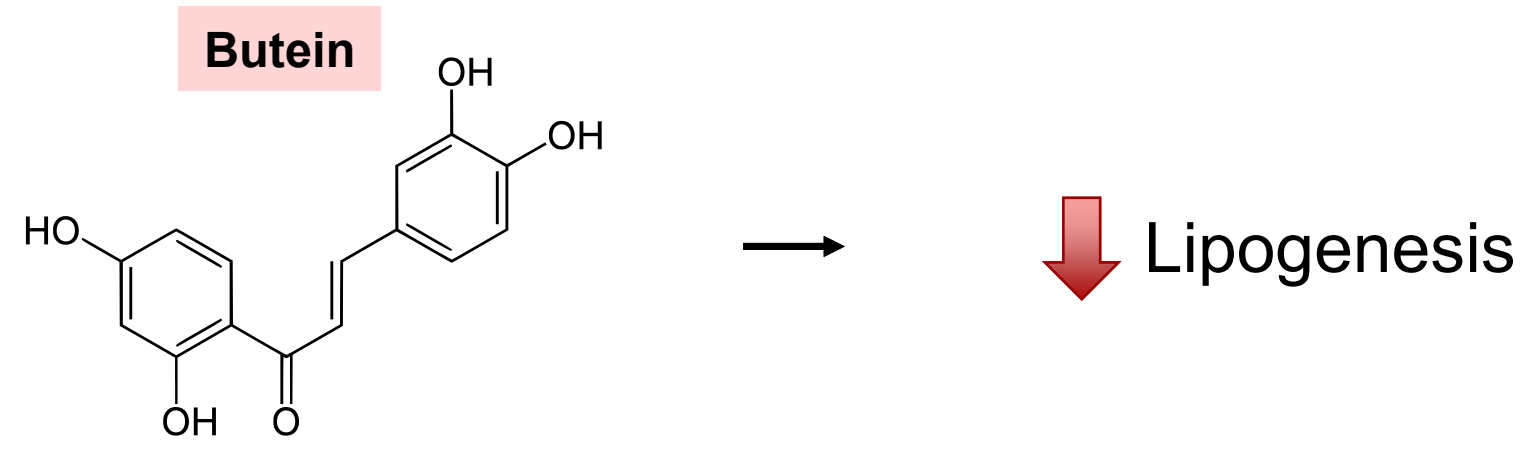

